\title{
Regularized minimum class variance extreme learning machine for language recognition
}

\author{
Jiaming $\mathrm{Xu}^{1,2^{*}}$, Wei-Qiang Zhang ${ }^{3}$, Jia Liu ${ }^{3}$ and Shanhong Xia ${ }^{1}$
}

\begin{abstract}
Support vector machines (SVMs) have played an important role in the state-of-the-art language recognition systems. The recently developed extreme learning machine (ELM) tends to have better scalability and achieve similar or much better generalization performance at much faster learning speed than traditional SVM. Inspired by the excellent feature of ELM, in this paper, we propose a novel method called regularized minimum class variance extreme learning machine (RMCVELM) for language recognition. The RMCVELM aims at minimizing empirical risk, structural risk, and the intra-class variance of the training data in the decision space simultaneously. The proposed method, which is computationally inexpensive compared to SVM, suggests a new classifier for language recognition and is evaluated on the 2009 National Institute of Standards and Technology (NIST) language recognition evaluation (LRE). Experimental results show that the proposed RMCVELM obtains much better performance than SVM. In addition, the RMCVELM can also be applied to the popular i-vector space and get comparable results to the existing scoring methods.
\end{abstract}

Keywords: Language recognition; Extreme learning machine; Single-hidden layer feedforward neural networks; Support vector machine

\section{Introduction}

The task of spoken language recognition is to automatically determine the identity of the language spoken in a speech sample [1]. It has received increasing attention due to its importance for the enhancement of automatic speech recognition (ASR) [2] and multi-language translation systems $[3,4]$.

The most popular language recognition techniques can usually be categorized as phonotactic [5-7] and acoustic approaches $[8,9]$. The former method is based upon phone recognizer followed by language models (PRLM), parallel PRLM (PPRLM), and supper vector machines PRLM. The later approach uses shifted-delta-cepstral (SDC) coefficients as a means of incorporating temporal information about the speech into the feature vector.

The introduction of support vector machines (SVMs) into language recognition resulted in significant improvement in performance [10]. SVMs have proven to be an effective method and have been widely used for many

\footnotetext{
*Correspondence: xujiaming09@gmail.com

${ }^{1}$ State Key Laboratory of Transducer Technology, Institute of Electronics, Chinese Academy of Sciences, 100190 Beijing, China

2 University of Chinese Academy of Sciences, 100190 Beijing, China Full list of author information is available at the end of the article
}

years [11]. SVMs perform a nonlinear mapping from an input space to an SVM feature space. The standard optimization method is then used to find the solution of maximizing the separating margin of two different classes in this potentially high-dimensional feature space while minimizing the training errors. In language recognition, we usually adopt the one-versus-rest tactic to deal with multiclass classification problems when using SVMs, namely, setting data from target language as the positive samples and data from all the other languages as the negative samples. In this way, the amount of training samples between the positive language and negative languages is unbalanced and sometimes it is even impossible to separate them properly by a linear hyperplane. In addition, the training of SVMs involves a quadratic programming (QP) problem, the computational complexity of SVM training algorithms is usually intensive, which is at least quadratic with respect to the number of training samples.

In recent years, extreme learning machine (ELM) $[12,13]$ has emerged and attracted the attention from more and more researchers. ELM is a single-hidden layer feedforward network (SLFN) which randomly selects input weights and hidden neuron biases without training.

\section{至 Springer}


The output weights are analytically determined by MoorePenrose generalized inverse. The input weights are the weights of the connections between input neurons and hidden neurons and the output weights are the weights of the connections between hidden neurons and output neurons. ELM overcomes the proposed challenges faced by SVMs. Solving the regularized least square problem in ELM is faster than solving the quadratic programming problem in standard SVMs. Recent papers show that predicting accuracy achieved by ELM is comparable with or even higher than that of SVMs [14, 15]. Besides, ELM can learn the training data not only one-by-one but also chunk-by-chunk [16], so it provides a promising method to deal with big data. Moreover, traditional kernel methods can also be applied to ELM [15]. Due to its excellent features, ELM has been successfully used in many areas and there has been increasing research interest in it [17-19]. Many researchers have came up with some methods to improve ELM theories, such as ELMs for noisy/missing data $[20,21]$ and imbalanced data [22]. In [23], minimum class variance extreme learning machine (MCVELM) was proposed for human action recognition and achieved excellent performance. MCVELM borrows the idea from minimum class variance support vector machines (MCVSVM) [24] which is inspired from the optimization of Fisher's discriminant ratio. The objective of MCVELM is to minimize both the intra-class variance of training data in the decision space and the training errors. However, the MCVELM does not take the structural risk into consideration, so it can lead to the over-fitting problem.

Inspired by its successful application to classification problems, in this paper, we introduce ELM into spoken language recognition, hoping to open up a new research direction for language recognition community. A new method referred to as regularized minimum class variance extreme learning machine (RMCVELM) is proposed and used as classifier for language recognition. Different from MCVELM, the proposed RMCVELM also minimizes the output weight norm directly which can effectively prevent the over-fitting problem. Therefore, the proposed RMCVELM can be considered as minimizing empirical risk, structural risk, and the intra-class variance of the training data in the decision space simultaneously. Since RMCVELM is based on structural risk minimization, it can be expected to provide better generalization ability.

To evaluate the effectiveness of the proposed RMCVELM, in this work, two types of acoustic features are used, GMM supervectors (GSVs) [25] and i-vectors [26]. The GSV has been widely used for many years and the i-vector approach, which provides an elegant way to capture the majority of useful variabilities of an utterance, has proven to be one of the most successful methods for language recognition nowadays $[27,28]$.
The outline of the paper is as follows. In Section 2, we introduce the ELM algorithm briefly. The proposed RMCVELM is described in detail in Section 3. In Section 4, experimental setup is introduced. In Section 5, we demonstrate the potential of the algorithm by applying it to language recognition task. In Section 6, computational complexity is analyzed. Finally, some conclusions are given in Section 7.

\section{Overview of extreme learning machine}

\subsection{Basic extreme learning machine}

ELM was first proposed by Huang [12] as an extremely simple and efficient method to train the single-hidden layer feedforward network. He has proven that the SLFN network with randomly chosen input weights and hidden neuron biases can approximate any continuous function to any desirable accuracy [29]. The input weights and hidden biases need not be tuned after randomly selected, and the output weights are analytically calculated using Moore-Penrose generalized pseudo-inverse.

Let us denote the training data as $\aleph=\left\{\left(\mathbf{x}_{i}, \mathbf{t}_{i}\right) \mid \mathbf{x}_{i} \in \mathbf{R}^{d}\right.$, $i=1, \ldots, N\}$, where $d$ is the dimension of training data and $N$ is the number of training data. $\mathbf{t}_{i}$ is the label of $\mathbf{x}_{i}$ which is a vector of length $m, m$ is the number of classes. If $\mathbf{x}_{i}$ belongs to class $j$, then all elements of $\mathbf{t}_{i}$ are zero except the $j$ th element, which takes the value 1 .

The structure of ELM is shown in Fig. 1. $L$ is the number of hidden nodes. $\mathbf{w}_{j}$ and $b_{j}$ are the input weights and bias of the $j$ th hidden node, respectively. Hidden node parameters $\mathbf{w}_{j}$ and $b_{j}$ remain fixed after randomly generated. $\boldsymbol{\beta}_{j}$ is the output weights of the $j$ th hidden node. The output of hidden layer with respect to training data $\mathbf{x}_{i}$ is $\mathbf{h}\left(\mathbf{x}_{i}\right)$,

$$
\mathbf{h}\left(\mathbf{x}_{i}\right)=\left[\boldsymbol{g}\left(\mathbf{w}_{1}^{T} \mathbf{x}_{i}+b_{1}\right) \ldots g\left(\mathbf{w}_{L}^{T} \mathbf{x}_{i}+b_{L}\right)\right]
$$

where $\boldsymbol{g}\left(\mathbf{w}_{j}^{T} \mathbf{x}_{i}+b_{j}\right)$ is the activation function of the $j$ th hidden node. Many activation function can be used, such as sigmod, sine, and RBFs. The most widely used is the sigmod function.

$$
\boldsymbol{g}\left(\mathbf{w}_{j}^{T} \mathbf{x}_{i}+b_{j}\right)=\frac{1}{1+\exp \left(-\left(\mathbf{w}_{j}^{T} \mathbf{x}_{i}+b_{j}\right)\right)}
$$

From the perspective of ELM, $\mathbf{h}\left(\mathbf{x}_{i}\right)$ is a feature map which transforms the sample $\mathbf{x}_{i}$ into ELM feature space.

The hidden layer output matrix $\mathbf{H}$ is defined as:

$$
\mathbf{H}=\left[\begin{array}{ccc}
\boldsymbol{g}\left(\mathbf{w}_{1}^{T} \mathbf{x}_{1}+b_{1}\right) & \ldots g\left(\mathbf{w}_{L}^{T} \mathbf{x}_{1}+b_{L}\right) \\
\vdots & \ldots & \vdots \\
\boldsymbol{g}\left(\mathbf{w}_{1}^{T} \mathbf{x}_{N}+b_{1}\right) & \ldots g\left(\mathbf{w}_{L}^{T} \mathbf{x}_{N}+b_{L}\right)
\end{array}\right]_{N \times L}
$$

the $j$ th column of $\mathbf{H}$ is the $j$ th hidden node output with respect to inputs $\mathbf{x}_{1}, \ldots, \mathbf{x}_{N}$. 


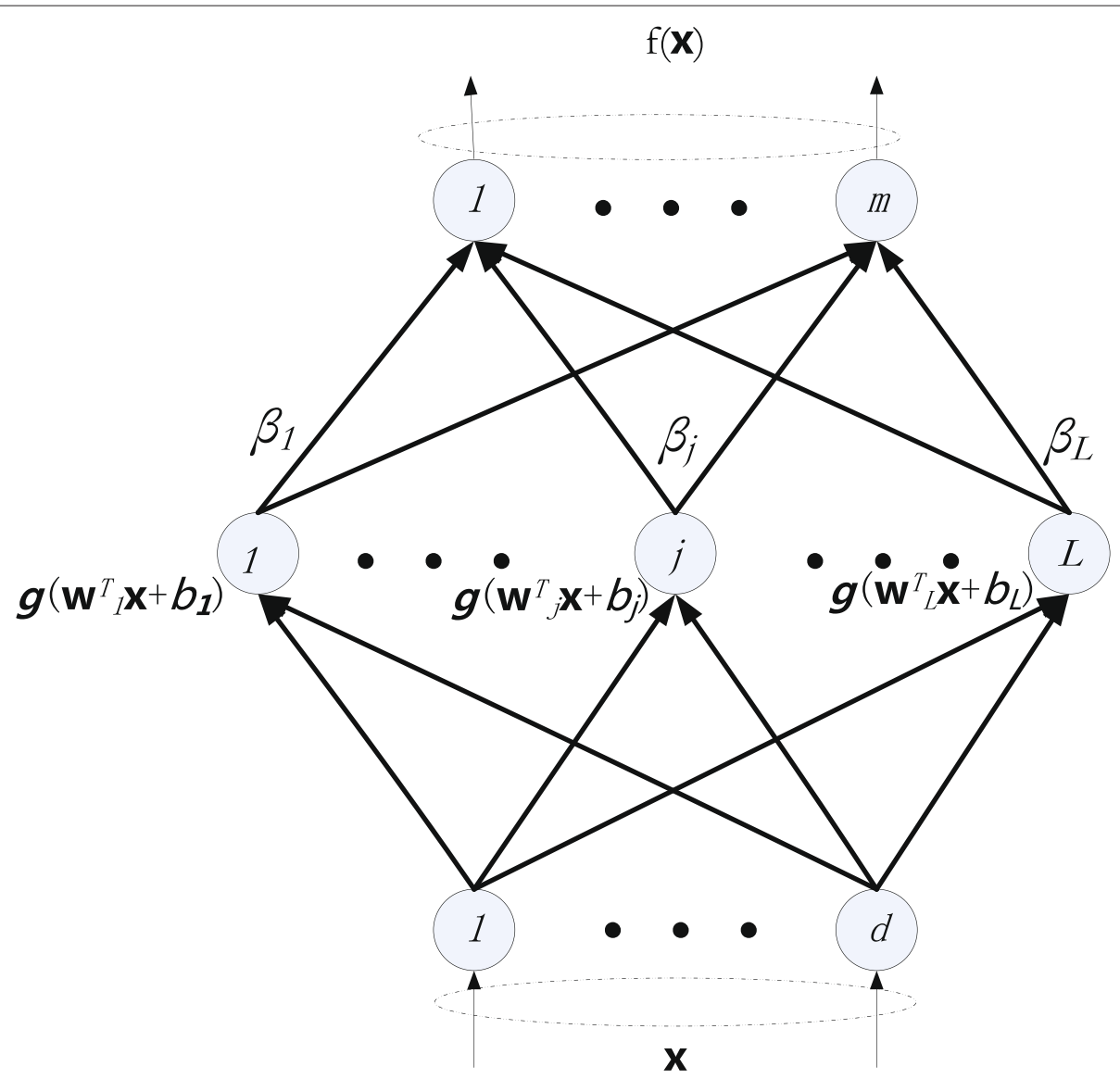

Fig. 1 Single-hidden layer feedforward network

The target of original ELM is to minimize the training error, namely

$$
\text { minimize : }\|\mathbf{H} \boldsymbol{\beta}-\mathbf{T}\|_{2}^{2}
$$

where

$$
\boldsymbol{\beta}=\left[\begin{array}{c}
\boldsymbol{\beta}_{1}^{T} \\
\vdots \\
\boldsymbol{\beta}_{L}^{T}
\end{array}\right]_{L \times m} \mathbf{T}=\left[\begin{array}{c}
\mathbf{t}_{1}^{T} \\
\vdots \\
\mathbf{t}_{N}^{T}
\end{array}\right]_{N \times m}
$$

The solution of the above linear system is:

$$
\boldsymbol{\beta}=\mathbf{H}^{\dagger} \mathbf{T}
$$

The orthogonal projection method can be efficiently used in ELM, namely $\mathbf{H}^{\dagger}=\left(\mathbf{H}^{T} \mathbf{H}\right)^{-1} \mathbf{H}^{T}$ if $\mathbf{H}^{T} \mathbf{H}$ is nonsingular or $\mathbf{H}^{\dagger}=\mathbf{H}^{T}\left(\mathbf{H} \mathbf{H}^{T}\right)^{-1}$ if $\mathbf{H} \mathbf{H}^{T}$ is nonsingular.

During the test stage, a test data $\mathbf{x}$ is scored using the following equation,

$$
\mathbf{f}(\mathbf{x})=\mathbf{h}(\mathbf{x}) \boldsymbol{\beta}=\mathbf{h}(\mathbf{x})\left(\mathbf{H}^{T} \mathbf{H}\right)^{-1} \mathbf{H}^{T} \mathbf{T}
$$

or

$$
\mathbf{f}(\mathbf{x})=\mathbf{h}(\mathbf{x}) \boldsymbol{\beta}=\mathbf{h}(\mathbf{x}) \mathbf{H}^{T}\left(\mathbf{H} \mathbf{H}^{T}\right)^{-1} \mathbf{T}
$$

\subsection{Regularized extreme learning machine}

The basic ELM described previously directly uses the normal equations for the least squares problem, so it can be considered as based on empirical risk minimization and tends to have over-fitting problems. Adding a regularization term to the error function can control over-fitting [30]. Based on this idea, the objective function of regularized extreme learning machine (RELM) can be written as,

$$
\text { Minimize : }\|\mathbf{H} \boldsymbol{\beta}-\mathbf{T}\|_{2}^{2}+\frac{C}{2}\|\boldsymbol{\beta}\|_{2}^{2}
$$

where $C$ is the user specified parameter and provides a tradeoff between the training error and the regularization term $\|\boldsymbol{\beta}\|_{2}^{2}$. Similar to ELM, the solution to RELM can be written as,

$$
\boldsymbol{\beta}=\left(\mathbf{H}^{T} \mathbf{H}+C \mathbf{I}\right)^{-1} \mathbf{H}^{T} \mathbf{T}
$$

In [13], Huang has discussed the similarities between RELM and SVM and proven that to minimize the norm of the output weight $\|\boldsymbol{\beta}\|_{2}$ is actually to maximize the distance of the separating margins of two different classes in 
the ELM feature space, which is similar to SVM. This conclusion is quite important and will be further discussed when introducing the RMCVELM.

\section{Regularized minimum class variance extreme learning machine}

Based on the previous introduction, in this section, we will describe RMCVELM in detail. We will first introduce the MCVELM and show some of it advantages when dealing with classification problems under certain conditions. Then the drawback of this algorithm is illustrated and RMCVELM is proposed to overcome the challenge faced by MCVELM.

\subsection{Minimum class variance extreme learning machine} In [23], minimum class variance extreme learning machine (MCVELM) was proposed for human action recognition. MCVELM borrows the idea from minimum class variance support vector machines (MCVSVM) [24] which is inspired from the optimization of Fisher's discriminant ratio. Instead of finding the maximum separating margin, MCVSVM aims at finding a hyperplane along which the intra-class variance of the training data is minimum. Similar to MCVSVM, the objective of MCVELM is to minimize both the intra-class variance of training data in the decision space and the training errors. The objective function of RMCVELM has the form

$$
\begin{aligned}
& \text { Minimize }: \frac{1}{2} \sum_{i=1}^{N}\left\|\boldsymbol{\xi}_{i}\right\|_{2}^{2}+\frac{C}{2} \operatorname{tr}\left(\boldsymbol{\beta}^{T} \mathbf{S}_{w} \boldsymbol{\beta}\right) \\
& \text { Subject to }: \mathbf{h}\left(\mathbf{x}_{i}\right) \boldsymbol{\beta}=\mathbf{t}_{i}^{T}-\boldsymbol{\xi}_{i}^{T}, i=1, \ldots, N
\end{aligned}
$$

where $\operatorname{tr}()$ is the trace of a matrix and $\xi_{i}=\left[\xi_{i, 1}, \ldots, \xi_{i, m}\right]^{T}$ is the training error vector of the $m$ output nodes with respect to the training sample $\mathbf{x}_{i} . \mathbf{S}_{w}$ is the within-class scatter matrix of the training samples in the ELM space defined by

$$
\mathbf{S}_{w}=\sum_{k=1}^{m} \sum_{i \in C_{k}}\left(\mathbf{h}\left(\mathbf{x}_{i}\right)-\mathbf{m}_{k}\right)\left(\mathbf{h}\left(\mathbf{x}_{i}\right)-\mathbf{m}_{k}\right)^{T}
$$

where $N_{k}$ is the number of training samples in the class $k$ and $\mathbf{m}_{k}=\frac{1}{N_{k}} \sum_{i=1}^{N_{k}} \mathbf{h}\left(\mathbf{x}_{i}\right)$ is the mean vector of class $k$. So the term $\operatorname{tr}\left(\boldsymbol{\beta}^{T} \mathbf{S}_{w} \boldsymbol{\beta}\right)$ represents intra-class variance of all the training samples in the decision space. The solution to MCVELM can be written as

$$
\boldsymbol{\beta}=\left(\mathbf{H}^{T} \mathbf{H}+C \mathbf{S}_{w}\right)^{-1} \mathbf{H}^{T} \mathbf{T}
$$

Compared with RELM, MCVELM takes the distribution of all the training data in the ELM feature space into consideration, not only the data near the separating margin. From Fig. 2, we can easily find the difference between RELM and MCVELM. In Fig. 2, some synthetic data are used to simulate samples in the ELM feature space. Two

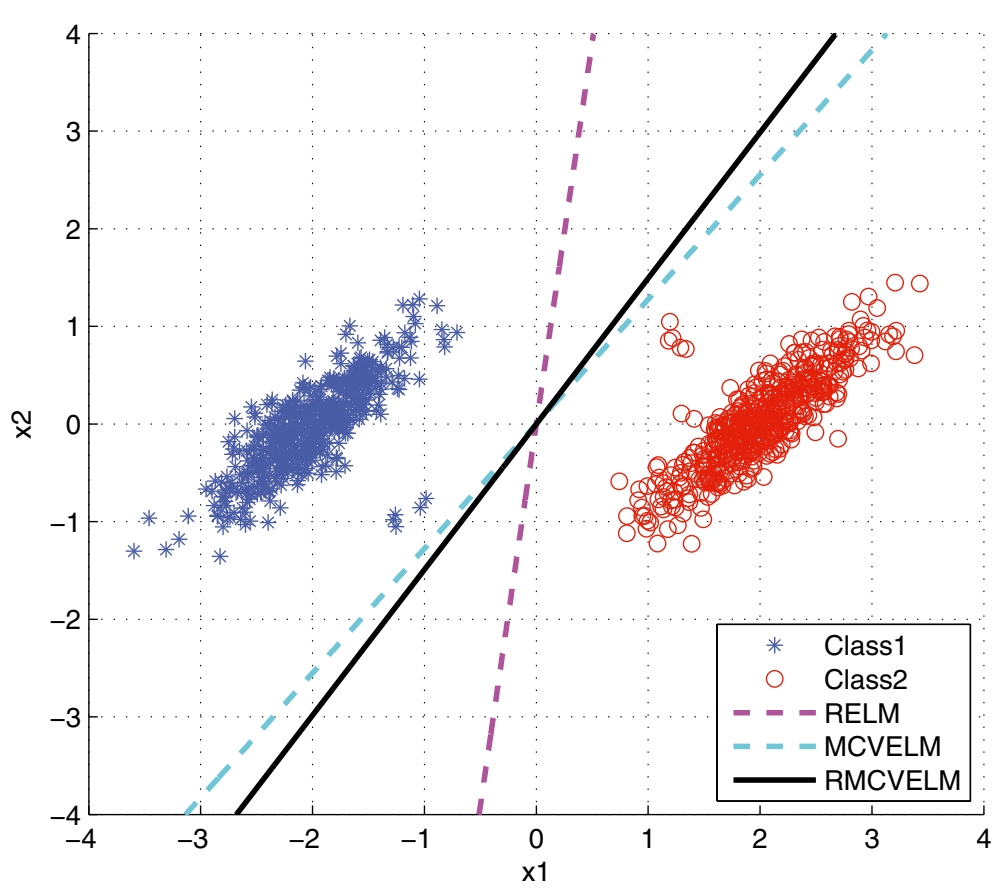

Fig. 2 Illustration of RELM and MCVELM optimization problems in the ELM space 
classes of Gaussian distribution data are randomly generated and several samples are added near the margin between the two classes. Then RELM and MCVELM are used to classify the data. As shown in Fig. 2, RELM tries to find a maximum separating margin, but this separating hyperplane can not effectively describe the data distribution so it loses some information which is helpful to classification. In this case, MCVELM can find a more rational separating hyperplane which is more consistent with the data distribution.

\subsection{Regularized minimum class variance extreme learning machine}

From previous analysis, we can see that MCVELM, which tries to find a separating hyperplane along which the intraclass variance is minimum can work better than RELM under certain conditions; however, there will be a serious problem when the samples are distributed as shown in Fig. 3. From Fig. 3a-d, the mean of each Gaussian is changed gradually along the $\mathrm{x} 2$-axis. Here we denote the mean vector of Class 1 as $\mu 1$ and the mean vector of Class 2 as $\mu 2 . \mu 1$ and $\mu 2$ in the four sub-figures are shown in Table 1. In this case, it is obvious that the hyperplane based on MCVELM can not separate the two classes properly. When there are slight changes in the relative position between the two classes, the separating hyperplane obtained by MCVELM varies greatly, as shown in Fig. $3 \mathrm{~b}-\mathrm{d}$, it rotates clockwise greatly. Besides minimizing training error, MCVELM always tries to find a separating line along which the intra-class variance is minimum, while the samples near the margin which can provide more important classification information are neglected.
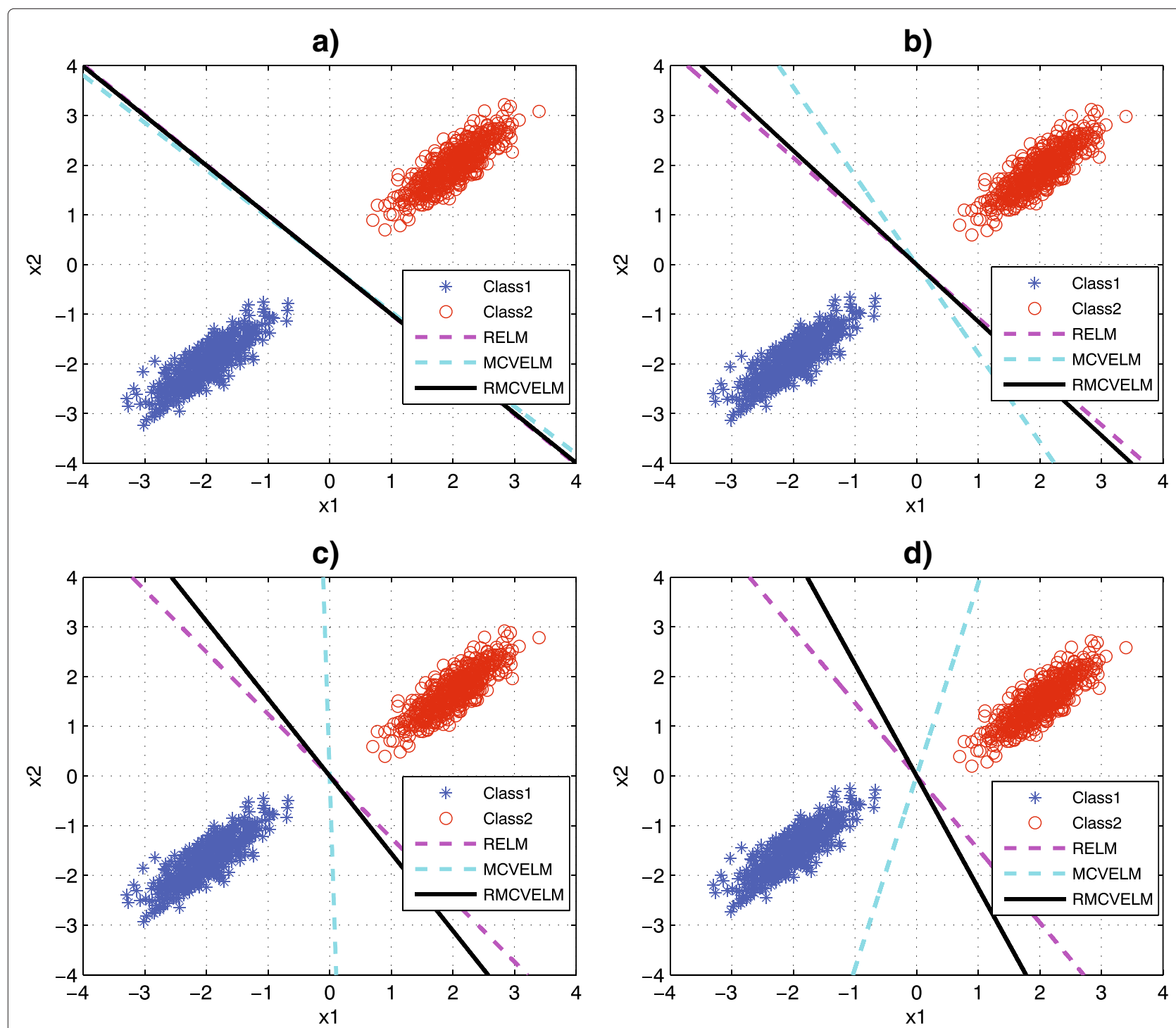

Fig. 3 a-d Illustration of RELM and MCVELM optimization problems in the ELM space 
Table 1 Mean vectors in Fig. 3

\begin{tabular}{lllll}
\hline Fig. 3 & $\mathrm{a}$ & $\mathrm{b}$ & $\mathrm{c}$ & $\mathrm{d}$ \\
\hline $\boldsymbol{\mu} 1$ & {$[-2,-2]$} & {$[-2,-1.9]$} & {$[-2,-1.7]$} & {$[-2,-1.5]$} \\
$\boldsymbol{\mu 2}$ & {$[2,2]$} & {$[2,1.9]$} & {$[2,1.7]$} & {$[2,1.5]$} \\
\hline
\end{tabular}

This objective makes the MCVELM too sensitive to the changes in the relative position between the two classes, even when the changes are not so big. So MCVELM tends to find separating hyperplanes as shown in Fig. 3. In this case, the samples from the two classes tend to overlap if they are projected onto the direction onto which the intra-class variance of training samples is minimum. The RELM, on the contrary, which aims at finding the maximum separating margin is not so sensitive to the slight position changes. Its separating hyperplane only rotates clockwise slightly. Under this condition, the samples near the margin can provide much more important information for classification, so RELM can work better than MCVELM. Therefore, the maximum margin term is quite important and should be taken into consideration.

From another point of view, as stated in Section 2.2, regularization term $\|\boldsymbol{\beta}\|_{2}^{2}$ can also be considered as structural risk. According to statistical learning theory, the real prediction risk of learning is consisted of empirical risk and structural risk. A model with good generalization ability should have the best trade-off between the two risks. However, MCVELM does not take the structural risk into consideration. Therefore MCVELM can not always achieve good generalization performance.

From the previous analysis, we can see that under certain conditions RELM can work quite better than MCVELM and vice versa. Each algorithm has its own advantages and drawbacks and no algorithm can work well under all conditions. However, the differences between the two algorithms also suggest that RELM and MCVELM could be complementary to each other. That means a combination of the two algorithms can provide a more robust classification algorithm. Based on this idea, in this work, we propose the regularized minimum class variance extreme learning machine (RMCVELM) which minimizes the empirical risk, structural risk, and intraclass variance of training data in the decision space simultaneously. Different from MCVELM, the output weight norm is minimized directly. The objective function of RMCVELM has the form

Minimize $: \frac{1}{2} \sum_{i=1}^{N}\left\|\boldsymbol{\xi}_{i}\right\|_{2}^{2}+\frac{C_{1}}{2}\|\boldsymbol{\beta}\|_{2}^{2}+\frac{C_{2}}{2} \operatorname{tr}\left(\boldsymbol{\beta}^{T} \mathbf{S}_{w} \boldsymbol{\beta}\right)$

Subject to $: \mathbf{h}\left(\mathbf{x}_{i}\right) \boldsymbol{\beta}=\mathbf{t}_{i}^{T}-\boldsymbol{\xi}_{i}^{T}, i=1, \ldots, N$
As stated before, $\|\quad \beta\|_{2}^{2}$ is the regularization term which aims at finding maximum separating margin in the ELM space and preventing over-fitting problem and $\operatorname{tr}\left(\boldsymbol{\beta}^{T} \mathbf{S}_{w} \boldsymbol{\beta}\right)$ represents intra-class variance of all the training samples in the decision space.

By submitting the constraints into the objective function, we obtain the following equivalent unconstrained optimization problem,

Minimize $: \frac{1}{2}\|\mathbf{H} \boldsymbol{\beta}-\mathbf{T}\|_{2}^{2}+\frac{C_{1}}{2}\|\boldsymbol{\beta}\|_{2}^{2}+\frac{C_{2}}{2} \operatorname{tr}\left(\boldsymbol{\beta}^{T} \mathbf{S}_{w} \boldsymbol{\beta}\right)$

The gradient of this objective function with respect to $\boldsymbol{\beta}$ can be written as,

$$
\nabla=\mathbf{H}^{T}(\mathbf{H} \boldsymbol{\beta}-\mathbf{T})+C_{1} \boldsymbol{\beta}+C_{2} \mathbf{S}_{w} \boldsymbol{\beta}
$$

By setting the gradient to zero, we obtain the solution to the RMCVELM,

$$
\boldsymbol{\beta}=\left(\mathbf{H}^{T} \mathbf{H}+C_{1} \mathbf{I}+C_{2} \mathbf{S}_{w}\right)^{-1} \mathbf{H}^{T} \mathbf{T}
$$

During the test stage, a test data $\mathbf{x}$ is scored by the following equation,

$$
\mathbf{f}(\mathbf{x})=\mathbf{h}(\mathbf{x}) \boldsymbol{\beta}
$$

The RMCVELM algorithm is summarized as follows.

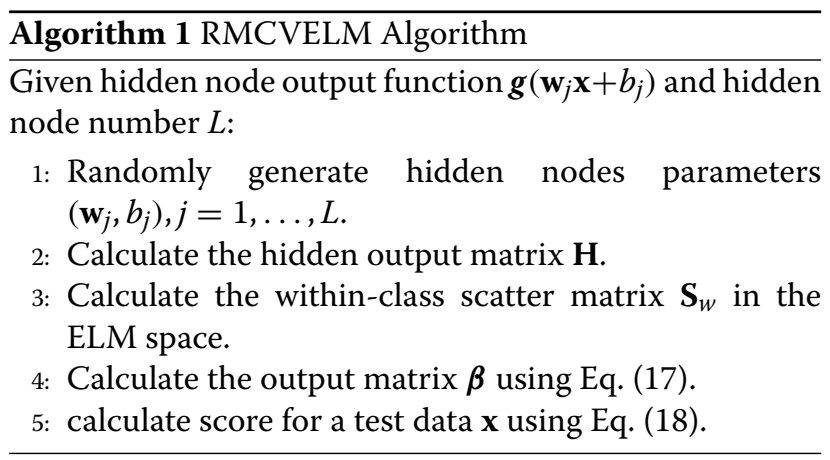

We should note that RMCVELM can be considered as a unified mode for basic ELM, RELM, and MCVELM. When $C_{1}=C_{2}=0$, RMCVELM is equivalent to basic ELM. If $C_{1}=0$ RMCVELM is equivalent to MCVELM and if $C_{2}=0$ RMCVELM is equivalent to RELM. From this point of view, RMCVELM takes advantage of the good features of ELM, RELM, and MCVELM, so it has the potential to deal with more complicated data and tends to achieve more robust performance. In Figs. 2 and 3, we also show the hyperplanes obtained by RMCVELM. It is easy to note that the hyperplanes found by RMCVELM are between the hyperplanes of RELM and MCVELM. In Fig. 2, RMCVELM can get a hyperplane which is consistent with the data distribution. In Fig. 3, the hyperplanes found by RMCVELM not only reflect the data distribution 
but also use the samples near the margin which play an important role in classification. So RMCVELM can work well in these two cases.

\section{Experimental setup}

\subsection{Training, development and evaluation data}

The experimental setup for this work is based on the NIST 2009 Language Recognition Evaluation (LRE) [31]. The 2009 LRE consisted of 23 linguistic classes. The 23 linguistic classes are Amharic, Bosnian, Cantonese, Creole, Croatian, Dari, English-American, English-Indian, Farsi, French, Georgian, Hausa, Hindi, Korean, Mandarin, Pashto, Portuguese, Russian, Spanish, Turkish, Ukrainian, Urdu, and Vietnamese. Both conversational telephone speech utterances (CTS) and narrow band telephone segments from Voice of America broadcasts (VOA) are used as data sources for the 23 linguistic classes.

The training data consists of both CTS and VOA data. The CTS samples come from previous NIST LRE (2007) and the CallFriend, CallHome, OGI-22 collections. The VOA data consist of narrow band segments from VOA broadcasts. We totally have about 30,000 samples for the whole training set.

The calibration back-end described in Section 4.3 was trained on development dataset, which comprises data from the dataset provided by NIST for the 2003, 2005, and 2007 LRE and VOA. Only data of the 23 target languages are used. This set has about 10,000 samples.

The evaluation set consists of all the data from the NIST 2009 LRE. Experiments are performed on the 23 languages closed-set task. The criterion for evaluation is pooled equal error rate (EER) and average cost $C_{\text {avg }}$ defined by NIST.

\subsection{Feature extraction}

For feature extraction, we first extract 13-dimensional MFCC features and the cepstral features are processed with RASTA filtering. Then SDCC features [8] are used with a 7-1-3-7 parameterization.

A language and gender independent UBM [32] is trained using all of the training data with eight iterations of EM adapting all parameters-means, mixture weights, and diagonal covariances. The number of mixture components is 512 .

After that, two different kinds of features are extracted for each utterance, namely GMM Supervectors (GSVs) [25] and i-vectors [26].

For the GSV extraction, a GMM is trained for each utterance, only means are adapted for GMM MAP training. The GSV for a given utterance is the stacked mean supervector which is normalized as follows,

$$
\widetilde{\mathbf{m}}_{i}=\sqrt{\lambda_{i}} \Sigma_{i}^{-1 / 2} \mathbf{m}_{i}
$$

where $\lambda_{i}, \boldsymbol{\Sigma}_{i}$ and $\mathbf{m}_{i}$ are the mixture weight, covariance and mean of the $i$ th GMM component. A 28,672 dimensional GSV is extracted for each utterance. The GSVs are then compensated using Nuisance Attribute Projection (NAP) [33].

The i-vectors framework has become very popular in speaker and language recognition. The main idea is that the GMM supervector (vector created by stacking all mean vectors from the GMM) for a given utterance can be modeled as follows,

$$
\mathbf{M}=\mathbf{m}+\mathbf{T w}
$$

where $\mathbf{m}$ is the language- and channel-independent mean supervector (which can be taken to be the UBM supervector), $\mathbf{T}$ is a rectangular matrix of low rank and the $\mathrm{i}$-vector $\mathbf{w}$ is a random vector having a standard normal distribution. For a given utterance, we use the standard method to extract the $\mathrm{i}$-vector as described in [26]. The dimension of each $\mathrm{i}$-vector is 400 .

\subsection{Calibration back-end}

The calibration back-end [34] uses the scores from the classifiers described above rather than the GSVs or the ivectors. It is trained on the separate development dataset described in Section 4.1 to obtain well-calibrated scores. Here, we use the MMI back-end [35] for calibration.

\section{Experimental result}

\subsection{GSV systems}

In order to evaluate the effectiveness of the proposed RMCVELM algorithm on language recognition performance, we first compare its performance with that of SVM, MCVELM, and RELM on the GSV features.

In our RMCVELM system, the input weight $\mathbf{w}_{i}$ is randomly generated between -0.5 and +0.5 , and $b_{i}$ is randomly generated between 0 and +1 . The number of hidden node is 20,000 . Here we use cross-validation technique on the training data to determine the parameters $C_{1}$ and $C_{2}$. The training data is partitioned into four groups. Then three of the groups are used to train a set of models that are then evaluated on the remaining group. This procedure is then repeated for all four possible choices for the groups, and the four performance scores are then averaged. According to this cross-validation technique, the optimal parameters are set to be $C_{1}=2000$ and $C_{2}=5$.

In the SVM system, we use the proposed one-versusrest strategy which is the standard method widely used for language recognition. The C value for SVM is set to be 0.8 , it is tuned by the cross-validation technique as stated in the RMCVELM system.

In the MCVELM system, $C_{1}$ is set to be 0 and $C_{2}=1.5$ which is achieved by the cross-validation technique stated above. Similarly, in the RELM system, $C_{2}$ is set to be 0 and $C_{1}=3000$. In the Basic ELM system, $C_{1}=C_{2}=0$. 
We also train a standard neural network (NN) with the same architecture as the ELM for comparison. The hidden node number is 120 and learning rate is 0.05 . Besides, dropout method is used, the dropout fraction is 0.8. In addition, the weight penalty term based on $L^{2}$ norm is also taken into consideration. The coefficient of the weight penalty term is 0.005 . We find that the dropout method and the weight penalty term must be used or there will be a serious over-fitting problem. All the above parameters are set by the cross-validation technique stated above.

Results for the various systems on GSV features are shown in Table 2.

First, we can easily note that the proposed RMCVELM outperforms SVM at all the three different durations. Especially, RMCVELM can greatly enhance the performance at the 30-s duration, obtaining at most $15 \%$ (EER) and $19 \%\left(C_{\text {avg }}\right)$ relative improvement compared with SVM, respectively. In addition, RMCVELM works quite better than MCVELM and RELM, which verifies our theory. RMCVELM is more robust compared with MCVELM and RELM.

We should also note that the NN is better than the basic ELM which has no regularization term and comparable to the RELM. This is because the regularization term plays an important role in preventing the over-fitting problem. However, the NN does not achieve better performance than RELM, one reasonable explanation is that the NN tends to have the local minima problem. The advantages of ELM compared to NN has been discussed in detail in [12] which shows that ELM can achieve better performance than $\mathrm{NN}$ in many applications.

\subsection{I-vector systems}

For the i-vector inputs, all the i-vectors are centered on the origin and then linear discriminant analysis(LDA) is used to reduce the dimension. Since we have 23 target languages, each i-vector is reduced to 22 dimensions. Each $\mathrm{i}$-vector is unit normalized after LDA, here we use $\mathbf{l}_{i}$ to denote the normalized i-vector after LDA for a given utterance $i$.

Table 2 Comparison of EERs (\%) and $C_{\text {avg }}$ for different training methods based on GSV at different durations for the 23 proposed languages of LREO9

\begin{tabular}{lllllllll}
\hline System & \multicolumn{1}{l}{ EER } & & & & $C_{\text {avg }}$ & & \\
\cline { 2 - 3 } & $30 \mathrm{~s}$ & $10 \mathrm{~s}$ & $3 \mathrm{~s}$ & & $30 \mathrm{~s}$ & $10 \mathrm{~s}$ & $3 \mathrm{~s}$ \\
\hline SVM & 3.21 & 8.77 & 21.88 & & 3.24 & 8.76 & 21.69 \\
ELM & 4.32 & 12.17 & 25.93 & & 4.30 & 12.46 & 26.13 \\
MCVELM & 4.12 & 11.58 & 26.17 & & 4.20 & 11.58 & 26.17 \\
RELM & 2.89 & 7.79 & 22.00 & & 2.82 & 7.86 & 21.97 \\
NN & 2.97 & 7.98 & 22.56 & & 2.95 & 7.97 & 22.56 \\
RMCVELM & 2.70 & 7.59 & 20.74 & & 2.62 & 7.60 & 20.62 \\
\hline
\end{tabular}

We first apply the cosine distance scoring (CDS) to score the i-vector, which has been widely used in language recognition [36].

$$
\text { score }_{l}=\mathbf{m}_{l}^{T} * \mathbf{1}_{\text {test }}
$$

where $\mathbf{m}_{l}$ is the model of language $l$,

$$
\mathbf{m}_{l}=\frac{\sum_{i=1}^{N_{l}} \mathbf{l}_{i}}{\left\|\sum_{i=1}^{N_{l}} \mathbf{l}_{i}\right\|_{2}}
$$

In addition, a widely used generative model called Gaussian back-end (GB) [28] is also used for comparison. In this case, i-vector distribution for each language is modeled by a Gaussian distribution, here a full covariance matrix is shared across all languages. During the test stage, each $\mathrm{i}$-vector $\mathbf{l}_{\text {test }}$ is scored as:

score $l=-\frac{1}{2} \mathbf{l}_{\text {test }}^{T} \boldsymbol{\Sigma}^{-1} \mathbf{l}_{\text {test }}+\mathbf{l}_{\text {test }}^{T} \boldsymbol{\Sigma}^{-1} \boldsymbol{\mu}_{l}-\frac{1}{2} \boldsymbol{\mu}_{l}^{T} \boldsymbol{\Sigma}^{-1} \boldsymbol{\mu}_{l}+$ const

where $\boldsymbol{\mu}_{l}$ is the mean vector for language $l$ and $\boldsymbol{\Sigma}$ is the covariance matrix.

Then SVM, NN, and RMCVELM are applied to the ivector. The $\mathrm{C}$ value for $\mathrm{SVM}$ is 1.2. For the NN, the hidden node number is 80 , and learning rate is 0.01 . The dropout fraction is 0.45 , and the coefficient of the $L^{2}$ norm weight penalty term is 0.006 . For the RMCVELE system, The number of hidden node is 3000 (experiments show that there is no significant improvement when the number of hidden node increases), and $C_{1}=2100, C_{2}=3$. All the parameters are determined as that in the GSV feature space.

The performance of the different methods are shown in Table 3. The results demonstrate that RMCVELM can achieve comparable performance to the widely used CDS and GB method. So it can also be successfully applied to the i-vector space.

In addition, we can easily note that the CDS and GB which are used as baselines can achieve similar performance. Here we think that the CDS can approximate the GB. Since all i-vectors are unit vectors and centered on the origin, the mean of the Gaussian distribution for each

Table 3 Comparison of EERs (\%) and $C_{\text {avg }}$ for different training methods based on i-vector at different durations for the 23 proposed languages of LRE09

\begin{tabular}{llllllll}
\hline System & \multicolumn{1}{l}{ EER } & \multicolumn{5}{c}{$C_{\text {avg }}$} \\
\cline { 2 - 3 } & $30 \mathrm{~s}$ & $10 \mathrm{~s}$ & $3 \mathrm{~s}$ & & $30 \mathrm{~s}$ & $10 \mathrm{~s}$ & $3 \mathrm{~s}$ \\
\hline CDS & 2.63 & 6.72 & 20.22 & & 2.59 & 6.80 & 20.36 \\
GB & 2.65 & 6.77 & 19.90 & & 2.60 & 6.86 & 20.02 \\
SVM & 2.70 & 6.78 & 19.92 & & 2.62 & 6.97 & 19.80 \\
NN & 2.95 & 7.87 & 21.32 & & 2.93 & 7.92 & 21.54 \\
RMCVELM & 2.65 & 6.67 & 19.65 & & 2.58 & 6.79 & 19.70 \\
\hline
\end{tabular}


language is approximately on the circle centered on the origin. The CDS averages i-vectors for a language, the model can roughly be regarded as a vector pointing from the origin to the mean of the Gaussian distribution for this language, so the separating margin between two classes is a linear hyperplane which is through the origin and equally divides the angle formed by the models of the two classes. The GB uses a shared covariance matrix, so the separating margin between two classes is also a linear hyperplane. If the covariance matrix is a diagonal matrix and the diagonal elements are the same, the GB hyperplane also will be through the origin and will be quite close to the CDS hyperplane. In this experiment, we find that the main diagonal elements of the full covariance matrix are much larger than other elements, so the full covariance matrix is close to a diagonal matrix. In addition, the main diagonal elements are close to each other. In this case, we can assume that the CDS is almost equivalent to the GB. So the two methods can achieve similar performance.

Besides, the NN can not get good performance as it is often very sensitive to many parameters, such as learning rate and dropout fraction, and tends to have the local minima problem.

\subsection{Discussion}

The experimental results show that in most cases, RMCVELM can achieve better performance than SVM. Compared with SVM, RMCVELM has some potential advantages.

In [13], the differences and similarities between SVM and ELM has been discussed. ELM and SVM have similar optimization formula. However, in SVM optimal
Lagrange multipliers $\alpha_{i}$ are found from the hyperplane $\sum_{i=1}^{N} \alpha_{i} t_{i}=0$, so SVM often obtains a suboptimal optimization. Different from SVM, ELM does not need to satisfy this condition, so ELM has milder optimization constraints and can find $\alpha_{i}$ from the entire space. RMCVELM, which is an extension of ELM, naturally inherits this good feature.

In addition, RMCVELM can be considered as a unified mode for ELM, regularized ELM, and MCVELM. It considers the training error, generalization ability, and distribution of all the training data in the ELM space simultaneously. By adjusting parameters $C_{1}$ and $C_{2}$, RMCVELM can learn much better even when the data distribution varies greatly. So it has the potential to deal with more complicated data. From this point of view, RMCVELM is more robust.

\section{Computational complexity}

In this section, the computational complexity is compared between SVM and RMCVELM. Training time for the two algorithms are shown in Fig. 4. We can note that the proposed RMCVELM is much faster than traditional SVM. The main computational cost for SVM comes from a QP problem, which is at least quadratic with respect to the number of training data. So the computational complexity of SVM is usually intensive when dealing with large problems. However, RMCVELM gets a solution based on Eq. (17), where the size of $\left(\mathbf{H}^{T} \mathbf{H}+C_{1} \mathbf{I}+C_{2} \mathbf{S}_{w}\right)$ is $L * L$. In most applications, the number of hidden node $L$ is much smaller than the number of training data: $L<<N$. So RMCVELM can greatly reduce the training time.

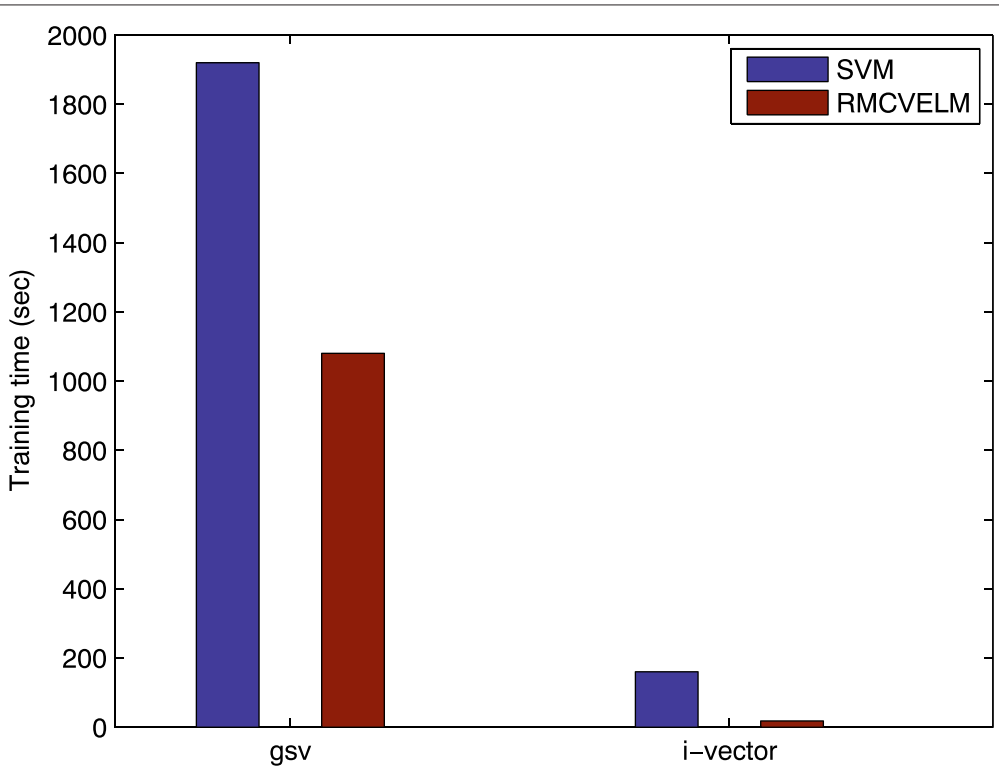

Fig. 4 Comparison of training time for SVM and RMCVELM on different features 


\section{Conclusions}

In this paper, we proposed a novel algorithm regularized minimum class variance extreme learning machine for language recognition. The RMCVELM aims at minimizing empirical risk, structural risk, and the intraclass variance of the training data in the decision space simultaneously. The proposed method improves the performance for language recognition and can learn at a much faster speed compared with traditional SVM. In addition, RMCVELM can also be applied to i-vector space and achieves an equivalent performance compared with the CDS and GB method. To our best knowledge, this is the first time such an approach is applied to language recognition.

\section{Competing interests}

The authors declare that they have no competing interests.

\section{Acknowledgements}

This work is supported by National Natural Science Foundation of China (Project 61370034, Project 61273268 and Project 61005019).

\section{Author details}

${ }^{1}$ State Key Laboratory of Transducer Technology, Institute of Electronics, Chinese Academy of Sciences, 100190 Beijing, China. ${ }^{2}$ University of Chinese Academy of Sciences, 100190 Beijing, China. ${ }^{3}$ National Laboratory for Information Science and Technology, Department of Electronic Engineering, Tsinghua University, 100084 Beijing, China.

Received: 25 November 2014 Accepted: 3 August 2015

Published online: 13 August 2015

\section{References}

1. H Li, B Ma, KA Lee, Spoken language recognition: from fundamentals to practice. Proc. IEEE. 101(5), 1136-1159 (2013)

2. F Biadsy, Automatic dialect and accent recognition and its application to speech recognition, PhD thesis, Columbia University (2011)

3. MA Zissman, KM Berkling, Automatic language identification. Speech Comm. 35(1), 115-124 (2001)

4. YK Muthusamy, E Barnard, RA Cole, Reviewing automatic language identification. Signal Proc. Mag. IEEE. 11(4), 33-41 (1994)

5. MA Zissman, Comparison of four approaches to automatic language identification of telephone speech. IEEE Transactions on Speech and Audio Processing. 4(1), 31 (1996)

6. WM Campbell, FS Richardson, DA Reynolds, in ICASSP (4). Language recognition with word lattices and support vector machines (IEEE, Honolulu, USA, 2007), pp. 989-992

7. W-Q Zhang, W-W Liu, Z-Y Li, Y-Z Shi, J Liu, Spoken language recognition based on gap-weighted subsequence kernels. Speech Communication. 60, 1-12 (2014)

8. PA Torres-Carrasquillo, E Singer, MA Kohler, RJ Greene, DA Reynolds, JR Deller Jr, in INTERSPEECH. Approaches to language identification using gaussian mixture models and shifted delta cepstral features (ISCA, Denver, USA, 2002)

9. W-Q Zhang, L He, Y Deng, J Liu, MT Johnson, Time-frequency cepstral features and heteroscedastic linear discriminant analysis for language recognition. IEEE Trans. Audio, Speech, and Lang. Process. 19(2), 266-276 (2011)

10. WM Campbell, E Singer, PA Torres-Carrasquillo, DA Reynolds, in ODYSSEY04-The Speaker and Language Recognition Workshop. Language recognition with support vector machines (ISCA, Toledo, Spain, 2004)

11. WM Campbell, JP Campbell, DA Reynolds, E Singer, PA Torres-Carrasquillo, Support vector machines for speaker and language recognition. Comput. Speech \& Lang. 20(2), 210-229 (2006)

12. G-B Huang, Q-Y Zhu, C-K Siew, in Neural Networks, 2004. Proceedings. 2004 IEEE International Joint Conference On. Extreme learning machine: a new learning scheme of feedforward neural networks, vol. 2 (IEEE, Budapest, Hungary, 2004), pp. 985-990

13. G-B Huang, DH Wang, Y Lan, Extreme learning machines: a survey. Int. J. Mach. Mach. Learn. Cybern. 2(2), 107-122 (2011)

14. G-B Huang, Q-Y Zhu, C-K Siew, Extreme learning machine: theory and applications. Neurocomputing. 70(1), 489-501 (2006)

15. G-B Huang, $\mathrm{H}$ Zhou, X Ding, R Zhang, Extreme learning machine for regression and multiclass classification. Systems, Man, and Cybernetics, Part B: Cybernetics, IEEE Transactions on. 42(2), 513-529 (2012)

16. N-Y Liang, G-B Huang, P Saratchandran, N Sundararajan, A fast and accurate online sequential learning algorithm for feedforward networks. Neural Netw. IEEE Trans. 17(6), 1411-1423 (2006)

17. J Xu, H Zhou, G-B Huang, in Information Fusion (FUSION), 2012 15th International Conference On. Extreme learning machine based fast object recognition (IEEE, Singapore, 2012), pp. 1490-1496

18. MM Sole, M Tsoeu, in AFRICON, 2011. Sign language recognition using the extreme learning machine (IEEE, Livingstone, Zambia, 2011), pp. 1-6

19. S Suresh, V Babu, N Sundararajan, in Control, Automation, Robotics and Vision, 2006. ICARCV'06. 9th International Conference On. Image quality measurement using sparse extreme learning machine classifier (IEEE, Singapore, 2006), pp. 1-6

20. P Horata, S Chiewchanwattana, K Sunat, Robust extreme learning machine. Neurocomputing. 102, 31-44 (2013)

21. Q Yu, Y Miche, E Eirola, M Van Heeswijk, E Séverin, A Lendasse, Regularized extreme learning machine for regression with missing data. Neurocomputing. 102, 45-51 (2013)

22. W Zong, G-B Huang, Y Chen, Weighted extreme learning machine for imbalance learning. Neurocomputing. 101, 229-242 (2013)

23. A losifidis, A Tefas, I Pitas, Minimum class variance extreme learning machine for human action recognition (2013)

24. S Zafeiriou, A Tefas, I Pitas, Minimum class variance support vector machines. Image Process. IEEE Trans. 16(10), 2551-2564 (2007)

25. WM Campbell, DE Sturim, DA Reynolds, Support vector machines using gmm supervectors for speaker verification. Signal Proc. Lett. IEEE. 13(5), 308-311 (2006)

26. N Dehak, P Kenny, R Dehak, P Dumouchel, P Ouellet, Front-end factor analysis for speaker verification. Audio, Speech, and Lang. Process, IEEE Trans. 19(4), 788-798 (2011)

27. N Dehak, PA Torres-Carrasquillo, D Reynolds, R Dehak, in INTERSPEECH 2011. Language Recognition via I-Vectors and Dimensionality Reduction (ISCA, Florence, Italy, 2011), pp. 857-860

28. D Martınez, O Plchot, L Burget, O Glembek, P Matejka, in Proceedings of Interspeech. Language recognition in ivectors space (Firenze, Italy, 2011), pp. 861-864

29. G-B Huang, Learning capability and storage capacity of two-hidden-laye feedforward networks. Neural Networks, IEEE Transactions on. 14(2), 274-281 (2003)

30. CM Bishop, Pattern Recognition and Machine Learning, vol. 1. (Springer, New York, 2006)

31. AF Martin, CS Greenberg, in Odyssey. The 2009 nist language recognition evaluation (ISCA, Brno, Czech, 2010), p. 30

32. DA Reynolds, TF Quatieri, RB Dunn, Speaker verification using adapted gaussian mixture models. Digital signal processing. 10(1), 19-41 (2000)

33. WM Campbell, DE Sturim, DA Reynolds, A Solomonoff, in Acoustics, Speech and Signal Processing, 2006. ICASSP 2006 Proceedings. 2006 IEEE International Conference On. Svm based speaker verification using a gmm supervector kernel and nap variability compensation, vol. 1 (IEEE, Toulouse, France, 2006)

34. M Penagarikano, A Varona, M Diez, $\sqcup$ Rodriguez-Fuentes, G Bordel, in INTERSPEECH. Study of different backends in a state-of-the-art language recognition system, (2012)

35. W ZHANG, T HOU, J LIU, Discriminative score fusion for language identification. Chin. J. Electron. 19(1), 124-128 (2010)

36. E Singer, P Torres-Carrasquillo, D Reynolds, A McCree, F Richardson, N Dehak, D Sturim, in Acoustics, Speech and Signal Processing, 2007. ICASSP 2007. IEEE International Conference On. The mitll nist Ire 2011 language recognition system (ISCA, Singapore, 2012), pp. 209-215 